\title{
PROJETO DE DINAMIZAÇÃO DA COLEÇÃO DE OBRAS RARAS DA BIBLIOTECA DO TRF 2a REGIÃO
}

\author{
OBRAS COLECCIÓN DE ESTIMULACIÓN \\ PROYECTO RARO TRF BIBLIOTECA SEGUNDO \\ REGIÓN
}

\begin{abstract}
Maria Cristina de Paiva Ribeiro - cristinapaiva40@yahoo.com.br Mestranda do Programa de Pós-graduação em Biblioteconomia (Mestrado Profissional) da Universidade Federal do Estado do Rio de Janeiro (UNIRIO). Bibliotecária do Tribunal Regional Federal da $2^{a}$ Região.
\end{abstract}

\begin{abstract}
RESUMO
Introdução: Uma biblioteca precisa manter um espaço com obras relevantes para o aprimoramento do conhecimento, onde serão organizadas e preservadas para a posteridade através de uma metodologia específica e com critérios eficazes de conservação. Esse é o intuito do Projeto de Dinamização da Organização e do Tratamento Técnico da Coleção de Obras Raras da Biblioteca do Tribunal Regional Federal da $2^{a}$ Região (TRF2).

Objetivo: Enfocar a importância da manutenção da Coleção de Obras Raras do TRF2, a partir de procedimentos necessários, através do Projeto.

Metodologia: Estudo descritivo-bibliográfico baseado em pesquisas bibliográficas para fundamentação teórica, em buscas na Internet das bibliotecas do Poder Judiciário que possuem acervos de obras raras e no estudo e análise do Projeto.

Resultados: O perfil de patrimônio cultural do livro raro está na ação do autor como agente da História e da Memória. A maioria das bibliotecas do Poder Judiciário possui acervos de obras raras, o que referenda a implantação do Projeto.

Conclusões: O Projeto possibilitou a elaboração de critérios norteadores de raridade bibliográfica, o estabelecimento de uma política de catalogação apropriada à descrição de livros raros e, colocou a necessidade da manutenção de um espaço para a perpetuação da memória do Direito através da produção jurídica representada na Coleção de Obras Raras.
\end{abstract}

Palavras-chave: Projeto de Dinamização da Organização e do Tratamento Técnico da Coleção de Obras Raras da Biblioteca do TRF 2a Região; Obra Rara; Biblioteca Jurídica; Organização do Conhecimento. 


\section{INTRODUÇÃO}

A Divisão de Biblioteca do Tribunal Regional Federal da $2^{a}$ Região iniciou a composição da sua Coleção de Obras Raras em 2001 através da aquisição, por compra, de livros raros da área jurídica, representativos da história do Direito em geral e no Brasil.

A continuidade do desenvolvimento da coleção de obras raras, incluindo a criação de uma Sala de Obras Raras, depende do aprimoramento epistemológico e técnico da equipe envolvida nesse trabalho. Para isso foi implantado o Projeto de Dinamização da Organização e do Tratamento Técnico da Coleção de Obras Raras.

A experiência inicial com a seleção e tratamento técnico do acervo ensejou a necessidade do aprimoramento da metodologia então utilizada. Questões como o estabelecimento de critérios bem fundamentados para embasar a seleção das obras, a atenção com o material entregue pelos livreiros, e a elaboração de um manual de boas práticas para viabilizar a catalogação preventiva dessas obras e seu cadastramento na base de dados gerenciada pelo Software Sophia, tornaram-se prementes no decorrer dos anos.

Para embasar o referido Projeto e destacar a necessidade da qualificação para a organização e preservação dessa coleção, foi feito um levantamento das bibliotecas que possuem acervos de livros raros jurídicos no âmbito do Poder Judiciário, em destaque na Justiça Federal.

\section{A DIVISÃO DE BIBLIOTECA DO TRIBUNAL REGIONAL FEDERAL DA $2^{\mathrm{a}}$ REGIÃO - DIBLI/TRF2}

A Biblioteca Afrânio Antonio da Costa está inserida na estrutura da Divisão de Biblioteca (DIBLI), da Secretaria de Documentação, Informação e Memória. Funciona no horário das $11 \mathrm{~h}$ às $19 \mathrm{~h}$. Mantém um espaço com equipamentos direcionados ao atendimento às pessoas com deficiência. Possui um acervo de cerca 45 mil volumes, especializado em Direito, formado pelas coleções de: Monografias; 
Obras de referência; Legislação (diários oficiais e coleções de leis); Periódicos, Bases de dados Jurídicas e a Coleção de obras raras. Os seus serviços são disponibilizados aos usuários internos do TRF 2 e externos. São os seguintes: Consultas ao acervo da Biblioteca; Empréstimos aos usuários internos e bibliotecas conveniadas; Pesquisas manuais (realizadas no acervo da Biblioteca) e/ou automatizadas (através de consulta aos bancos de dados locais e externos, através da Internet); Disseminação Seletiva da Informação; Intercâmbio - serviço cooperativo com outras Bibliotecas no âmbito da Justiça Federal e internacionalmente; Página eletrônica - disponibilidade de uma página na Intranet/Internet com os serviços disponíveis, catálogo eletrônico, bases de dados jurídicas, regulamento, boletim, etc.

Fornece os seguintes produtos: Revista Biblios: revista especializada em assuntos relacionados com o universo do livro jurídico, e o Boletim Biblios Digital.

\section{PROJETO DE DINAMIZAÇÃO DA ORGANIZAÇÃO E DO TRATAMENTO TÉCNICO DA COLEÇÃO DE OBRAS RARAS}

A Coleção de Obras Raras da DIBLI é formada por cerca de 1.400 (mil e quatrocentos) volumes adquiridos, em sua maioria, por compra e, também, por doação, incluindo as obras raras advindas da fusão das Bibliotecas do TRF $2^{a}$ Região e da Seção Judiciária do Estado do Rio de Janeiro - SJRJ.

O projeto de dinamização consiste na otimização das atividades de pesquisa, catalogação, classificação e indexação das obras, além da ampliação e adequação do espaço físico destinado ao armazenamento das obras raras, através da aquisição de estantes deslizantes próprias para a manutenção da salvaguarda do acervo, mobiliário apropriado à consulta e climatização adequada para a preservação e conservação das obras. 
O Projeto está inserido no Planejamento Estratégico da Justiça Federal, cujo objetivo estratégico consiste em agilizar os trâmites judiciais através da prestação jurisdicional mais ágil, mediante a implementação de ações que aumentem a produtividade de magistrados e servidores. Uma dessas ações é a prestação de serviços de qualidade na busca da satisfação dos usuários da Justiça Federal, de modo a assegurar os direitos de cidadania. O objetivo final é a celeridade na prestação jurisdicional. A disponibilidade do acervo da Coleção de Obras Raras da DIBLI ao público está dentro desse contexto em razão da importância de obras que representam a doutrina sedimentada do Direito (BRASIL, 2014).

\section{CRITÉRIOS DE SELEÇÃO DAS OBRAS RARAS}

O critério de raridade bibliográfica é necessário pelo fato de que tais livros carecem de tratamento diferenciado, visto o seu valor histórico, cultural, monetário e a dificuldade em obtê-los no mercado livreiro. $\mathrm{O}$ critério adotado pelas bibliotecas, geralmente está vinculado à antiguidade e ao valor histórico-cultural da obra.

Ainda não existe política nacional que oriente a identificação e qualificação de acervos raros, principalmente na área jurídica. Cada instituição elabora sua política mediante critérios utilizados por outras bibliotecas e os determinados pela Biblioteca Nacional.

A determinação dos critérios para a seleção das obras raras da DIBLI será baseada em alguns critérios utilizados por bibliotecas jurídicas como a Biblioteca do Ministério da Justiça, do Conselho da Justiça Federal, do Superior Tribunal de Justiça e da Faculdade de Direito da Universidade Federal do Rio de Janeiro - UFRJ.

Num primeiro estudo, chegamos aos seguintes critérios: livros impressos fora do Brasil até 1800; livros impressos no Brasil até 1860; primeiras edições (edições princeps); segundas edições até 1889; 
edições com tiragem aproximada de 300 exemplares; obras autografadas, com dedicatórias ou numeradas por autores renomados.

Os critérios estabelecidos não obstam a observação de outros critérios adotados pela Biblioteca Nacional/Rio de Janeiro.

\section{PROCESSAMENTO TÉCNICO DO ACERVO DE OBRAS RARAS}

A catalogação de obras raras não pode ser igual à catalogação de livros comuns. Assim como afirma Kakumori (2014), algumas bibliotecas procedem à catalogação das obras raras apenas as identificando no $\mathrm{n}^{\circ}$ de localização das mesmas na estante. Porém a catalogação é de suma importância para que os usuários tenham conhecimento das obras que existem ali. Há de ser necessário o destaque das características dos livros antigos. Para permitir a descrição de material bibliográfico existem o Código de Catalogação Anglo-Americano - AACR2, Descrição Bibliográfica Internacional Normalizada - ISBD e a Catalogação Descritiva de Livros Raros DCRM (b).

Apesar de existir o Manual de Catalogação Descritiva para Livros Raros - DCRM(b), uma variação do AACR2, que deve ser usado na descrição de todos os materiais publicados antes de 1801, na DIBLI utilizamos o ACCR2, onde qualquer desvio verificado na obra deve ser incluído em uma nota. O destaque citado por Kakumori (2014) é descrito nas notas porque outros elementos descritivos só pertencem às notas.

Existem as notas obrigatórias, onde são registrados qualquer desvio a partir de uma transcrição rigorosa de provas; a presença de errata; a presença de invólucros ou sobrecapas; declaração de assinaturas que aparecem no item; notas sobre a descrição de um trabalho (incluindo a descrição de ligação, impressão e tipologia) também são valiosas. Várias notas podem ser juntas em uma só, com 
diferentes elementos descritivos, separados por um ponto e vírgula. As Notas Locais são utilizadas para informações específicas do exemplar, tais como proveniência e tratamento de conservação e imperfeições.

De acordo com Rodrigues (2014), a catalogação de livros raros envolve, basicamente, três etapas:

- $\quad 1^{a}$ etapa: Análise bibliológica - consiste no levantamento de dados que servirá como ponto de partida para as próximas etapas: a pesquisa bibliográfica e a descrição bibliográfica. $\mathrm{Na}$ etapa da análise bibliológica, todas as características do exemplar a ser catalogado deverão ser observadas, incluindo: características bibliográficas: informações sobre autor(es), título, data de publicação, impressor, local de publicação, paginação, presença de licenças e/ou privilégios etc.; características físicas: ilustrações, gravuras, caracteres especiais, disposição do texto, marcas tipográficas etc.; características intrínsecas: falhas na paginação, presença de anotações manuscritas, marcas de propriedade (assinaturas, carimbos, ex-libris) etc.

- $\quad 2^{\mathrm{a}}$ etapa: Pesquisa bibliográfica - consiste na seleção de fontes bibliográficas para determinação de raridade e levantamento de informações sobre a história da obra e seu autor. Maiores informações em: Repertórios bibliográficos.

- $\quad 3^{a}$ etapa: Descrição bibliográfica - consiste em descrever as informações que representam cada obra e, complementarmente, o seu conteúdo.

6 BIBLIOTECAS DO PODER JUDICIÁRIO, COM ÊNFASE NA JUSTIÇA FEDERAL, QUE POSSUEM ACERVOS DE OBRAS RARAS

Batista (2010) destaca a grande importância do estudo dos livros como patrimônio cultural porque, segundo afirma, sejam eles antigos ou 
Maria Cristina de Paiva Ribeiro

Projeto de dinamização da coleção de obras raras da Biblioteca do TRF $2^{\circ}$ Região

raros, são considerados documentos representativos da memória de uma sociedade, onde deve ser considerado como patrimônio histórico e cultural, um patrimônio literário e intelectual de uma região, representativo da memória regional, nacional e mundial.

Para dar relevância ao perfil histórico-cultural das obras raras e referendar, junto à SED, o Projeto de Dinamização da Organização e do Tratamento Técnico da Coleção de Obras Raras, foi procedido um levantamento de todas as bibliotecas do Poder Judiciário que possuem acervos de obras raras, com destaque para as pertencentes à Justiça Federal.

Abaixo, no Quadro 1, estão dispostos a lista das bibliotecas arroladas na pesquisa, e o Gráfico 1, que apresenta estatisticamente a representatividade das coleções de obras raras no contexto do Poder Judiciário (federal e estadual). 
Maria Cristina de Paiva Ribeiro

Projeto de dinamização da coleção de obras raras da Biblioteca do TRF $2^{\circ}$ Região

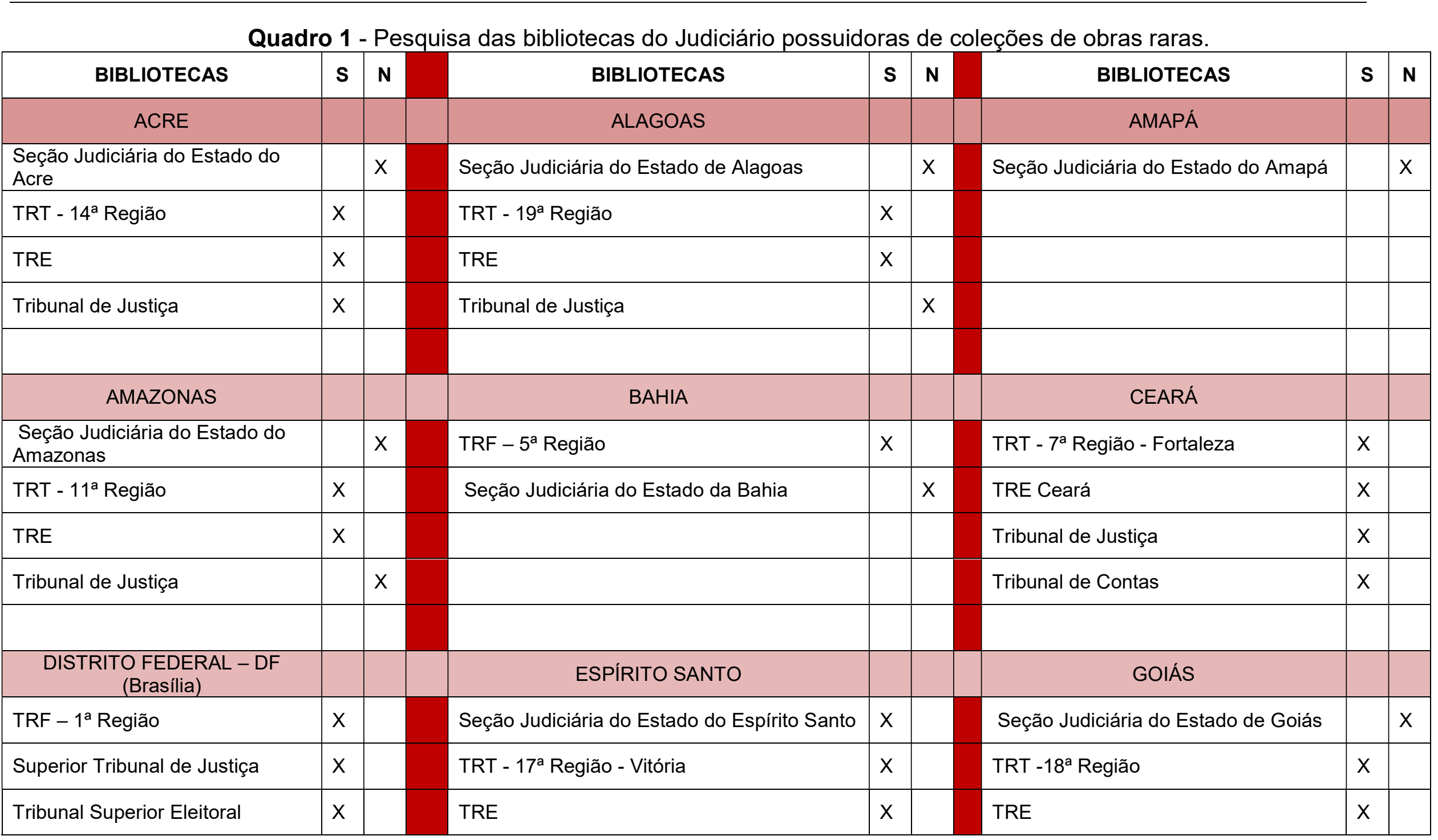

Inf. Prof., Londrina, v. 4, n. 1, p. 135 - 150, jan./jun. 2015. 
Maria Cristina de Paiva Ribeiro

Projeto de dinamização da coleção de obras raras da Biblioteca do TRF $2^{\circ}$ Região

\begin{tabular}{|c|c|c|c|c|c|c|c|c|}
\hline Seção Judiciária do DF & & $\mathrm{x}$ & Tribunal de Contas & & $\mathrm{x}$ & Tribunal de Contas & $\mathrm{x}$ & \\
\hline TRT - $10^{\mathrm{a}}$ Região & $\mathrm{x}$ & & Tribunal de Justiça & $\mathrm{x}$ & & Tribunal de Justiça & & $\mathrm{X}$ \\
\hline TRE & $\mathrm{x}$ & & & & & & & \\
\hline Tribunal de Contas & $\mathrm{x}$ & & & & & & & \\
\hline MARANHÃO & & & MATO GROSSO & & & MATO GROSSO DO SUL & & \\
\hline $\begin{array}{l}\text { Seção Judiciária do Estado do } \\
\text { Maranhão }\end{array}$ & & $\mathrm{x}$ & Seção Judiciária do Estado do Mato Grosso & & $\mathrm{x}$ & TRT - 24ª Região & $x$ & \\
\hline TRT - 16ª Região - São Luís & $\mathrm{x}$ & & TRT - 23a Região - Cuiabá & $\mathrm{X}$ & & TRE & $\mathrm{x}$ & \\
\hline TRE & $\mathrm{x}$ & & TRE & $\mathrm{x}$ & & Tribunal de Justiça & $x$ & \\
\hline Tribunal de Justiça & $\mathrm{x}$ & & Tribunal de Contas & $x$ & & & & \\
\hline Tribunal de Contas & & $\mathrm{x}$ & Tribunal de Justiça & & $\mathrm{X}$ & & & \\
\hline MINAS GERAIS & & & PARÁ & & & PARAÍBA & & \\
\hline $\begin{array}{l}\text { Seção Judiciária do Estado de } \\
\text { Minas Gerais }\end{array}$ & & $\mathrm{X}$ & Seção Judiciária do Estado do Pará & & $x$ & $\begin{array}{l}\text { Seção Judiciária do Estado da } \\
\text { Paraíba }\end{array}$ & & $x$ \\
\hline TRT - $3^{\text {a }}$ Região & $\mathrm{x}$ & & TRT - Belém do Pará & $x$ & & TRT - João Pessoa & $x$ & \\
\hline TRE - Minas Gerais & $x$ & & TRE & & & TRE & $x$ & \\
\hline Tribunal de Contas & $\mathrm{x}$ & & Tribunal de Justiça & $x$ & & Tribunal de Justiça & & $x$ \\
\hline Tribunal de Justiça & $x$ & & & & & Tribunal de Contas & $x$ & \\
\hline
\end{tabular}

Inf. Prof., Londrina, v. 4, n. 1, p. 135 - 150, jan./jun. 2015. 
Maria Cristina de Paiva Ribeiro

Projeto de dinamização da coleção de obras raras da Biblioteca do TRF $2^{\circ}$ Região

\begin{tabular}{|c|c|c|c|c|c|c|c|c|}
\hline PARANÁ & & & PERNAMBUCO & & & PIAUÍ & & \\
\hline $\begin{array}{l}\text { Seção Judiciária do Estado do } \\
\text { Paraná }\end{array}$ & & $\mathrm{x}$ & TRF - $5^{\mathrm{a}}$ Região - Recife & $x$ & & Seção Judiciária do Estado do Piauí & & $x$ \\
\hline TRT - 9a Região & $x$ & & Seção Judiciária do Estado de Pernambuco & & $x$ & TRT - 22ª Região & $\mathrm{x}$ & \\
\hline TRE & $\mathrm{x}$ & & TRE & $x$ & & TRE & $x$ & \\
\hline Tribunal de Contas & & $\mathrm{x}$ & Tribunal de Justiça & & $\mathrm{x}$ & Tribunal de Justiça & & $\mathrm{x}$ \\
\hline \multirow[t]{2}{*}{ Tribunal de Justiça } & $x$ & & Tribunal de Contas & $\mathrm{X}$ & & Tribunal de Contas & & $x$ \\
\hline & & & TRT - 6a Região & $\mathrm{X}$ & & & & \\
\hline RIO DE JANEIRO & & & RIO GRANDE DO NORTE & & & RIO GRANDE DO SUL & & \\
\hline TRF - $2^{\mathrm{a}}$ Região & $\mathrm{X}$ & & $\begin{array}{l}\text { Seção Judiciária do Estado do Rio Grande } \\
\text { do Norte }\end{array}$ & & $\mathrm{x}$ & TRF - $4^{\mathrm{a}}$ Região & $\mathrm{x}$ & \\
\hline TRT - 1a Região & $\mathrm{x}$ & & TRT - 21a Região & $\mathrm{x}$ & & $\begin{array}{l}\text { Seção Judiciária do Estado do Rio } \\
\text { Grande do Sul }\end{array}$ & & $\mathrm{x}$ \\
\hline TRE & $\mathrm{x}$ & & TRE & $x$ & & TRT - 4a Região - Porto Alegre & $x$ & \\
\hline Tribunal de Justiça & $x$ & & Tribunal de Contas & $x$ & & TRE & $x$ & \\
\hline \multirow[t]{2}{*}{ Tribunal de Contas } & & $x$ & Tribunal de Justiça & $x$ & & Tribunal de Justiça & $x$ & \\
\hline & $x$ & & & & & Tribunal de Contas & & $\mathrm{x}$ \\
\hline RONDÔNIA & & & RORAIMA & & & SANTA CATARINA & & \\
\hline Seção Judiciária de Rondônia & & $x$ & Seção Judiciária de Roraima & & $x$ & TRF - $4^{\mathrm{a}}$ Região & $x$ & \\
\hline TRT - 14ª Região & $x$ & & Tribunal de Justiça & & $x$ & $\begin{array}{l}\text { Seção Judiciária do Estado de Santa } \\
\text { Catarina }\end{array}$ & & $x$ \\
\hline
\end{tabular}

Inf. Prof., Londrina, v. 4, n. 1, p. 135 - 150, jan./jun. 2015. 
Maria Cristina de Paiva Ribeiro

Projeto de dinamização da coleção de obras raras da Biblioteca do TRF $2^{\circ}$ Região

\begin{tabular}{|c|c|c|c|c|c|c|c|c|}
\hline TRE & $\mathrm{x}$ & & TRT - $11^{\text {a }}$ Região & $x$ & & TRT - $12^{\mathrm{a}}$ Região & $\mathrm{X}$ & \\
\hline Tribunal de Justiça & & $\mathrm{x}$ & TRE & $x$ & & TRE & $\mathrm{x}$ & \\
\hline Tribunal de Contas & & $x$ & & & & Tribunal de Justiça & $\mathrm{x}$ & \\
\hline & & & & & & Tribunal de Contas & & $\mathrm{x}$ \\
\hline SÃO PAULO & & & SERGIPE & & & TOCANTINS & & \\
\hline TRF - $3^{\text {a }}$ Região & & $\mathrm{X}$ & Seção Judiciária do Estado de Sergipe & & $\mathrm{X}$ & Seção Judiciária do Tocantins & & $\mathrm{X}$ \\
\hline TRT - 15a Região - Campinas & $\bar{X}$ & & TRT - 20ª Região & $\bar{X}$ & & TRT - $10^{\mathrm{a}}$ Região & $\mathrm{X}$ & \\
\hline TRE & $\mathrm{X}$ & & TRE & & & TRE & $\mathrm{X}$ & \\
\hline TRT - $2^{\mathrm{a}}$ Região & $\bar{X}$ & & Tribunal de Justiça & & $\mathrm{X}$ & Tribunal de Justiça & & $\bar{x}$ \\
\hline Tribunal de Justiça & $\mathrm{X}$ & & Tribunal de Contas & & $x$ & Tribunal de Contas & & $\mathrm{X}$ \\
\hline Tribunal de Contas & $\mathrm{X}$ & & & & & & & \\
\hline
\end{tabular}

Fonte: A autora, 2015. 
Gráfico 1- Perfil estatístico do Quadro 1

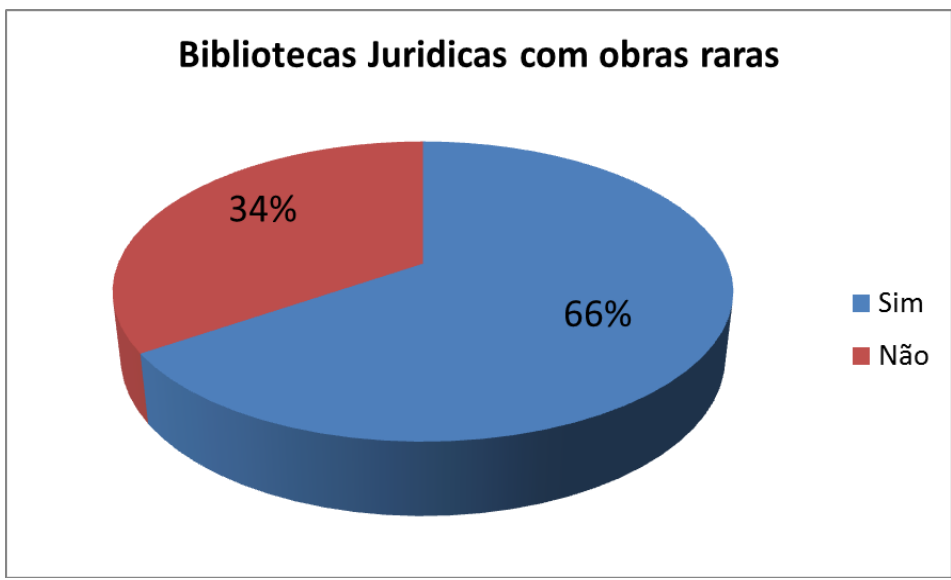

Fonte: A autora (2015).

\section{CONSIDERAÇÕES FINAIS}

A abordagem das obras raras como patrimônio cultural de um país, se faz verdadeira quando entendemos que os livros representam o conhecimento, cultura ou a história de um povo. As obras jurídicas têm o papel de representar o pensamento de grandes juristas e jurisconsultos que legaram a sua vida ao estudo do Direito e à aplicação da norma jurídica. O ideal de uma biblioteca, jurídica ou não, é manter um espaço onde essas obras relevantes para o aprimoramento do conhecimento, sejam organizadas e preservadas para a posteridade através de uma metodologia específica e com critérios eficazes de conservação, para que a memória da humanidade seja preservada através dos livros.

O Projeto de Dinamização da Organização e do Tratamento Técnico da Coleção de Obras Raras da DIBLI deu início à elaboração de critérios norteadores de raridade bibliográfica, ao estabelecimento de uma política de catalogação apropriada à descrição de livros raros e, principalmente, ao convencimento sobre a necessidade de manutenção de um espaço para a perpetuação da memória através da produção jurídica representada na Coleção de Obras Raras. Além de estar em conformidade com o Planejamento Estratégico da Justiça Federal, o que Ihe dá respaldo institucional. 
Maria Cristina de Paiva Ribeiro

Projeto de dinamização da coleção de obras raras da Biblioteca do TRF $2^{\circ}$

Região

\section{REFERÊNCIAS}

BATISTA, Aline Herbstrith. Estudo, catalogação e análise de obras raras da Biblioteca da Faculdade de Direito da UFPEL, datadas até 1840. In: SEMINÁRIO INTERNACIONAL EM MEMÓRIA E PATRIMÔNIO, 4., 2010, Pelotas/RS. Anais eletrônicos... $\quad$ Pelotas, $2010 . \quad$ Disponível em: <http://hdl.handle.net/123456789/225>. Acesso em: 30 jun. 2015.

BRASIL. Conselho da Justiça Federal. A estratégia da Justiça Federal: 2015/2020. Brasília: CJF, 2014. Anexo da Resolução CJF na 313/2014.

KAKUMORI, Bianca Adami Togo. Catalogação de obras raras: um estudo de caso. 52 p. Trabalho de Conclusão de Curso (Graduação em Biblioteconomia)Faculdade de Ciência da Informação, Universidade de Brasília, 2014.

RODRIGUES, Márcia Carvalho. Obras raras: catalogação. Blog Obras Raras, 2014. Disponível em: <https://obrasraras.wordpress.com/catalogacao/>. Acesso em: 25 jun. 2015.

\section{Title}

Works collection stimulation project of rare TRF library 2nd region

\section{Abstract}

Introduction: A library needs to keep a space with relevant works for the improvement of knowledge, which will be organized and preserved for posterity through a specific and effective conservation criteria methodology. This is the intention of the Organization Streamlining Project and Technical Treatment of Rare Books Collection Library Federal Regional Court of the 2nd Region (TRF2).

Objective: To focus the importance of maintaining the Rare Books Collection TRF2 from procedures required by the Project.

Methodology: Descriptive and bibliographic study based on bibliographic research to theoretical basis in searches on the Internet of the Judiciary libraries that hold collections of rare books and study and analysis of the Project.

Results: Cultural heritage profile of the rare book is in the author's action as an agent of history and memory. Most of the judicial branch libraries have collections of rare books, which reinforces the implementation of the Project.

Conclusions: The Project enabled the development of guiding criteria bibliographic rarity, the establishment of an appropriate cataloging policy to the description of rare books and placed the need to maintain a space for the perpetuation of the Law of memory by legal production represented in Rare Books collection.

Keywords: The Organization's Promotion of Design and Technical Treatment of the Rare Books Collection TRF 2nd Region Library; Rare Book; Law Library; Organization of Knowledge.

Inf. Prof., Londrina, v. 4, n. 1, p. 151- 164, jan./jun. 2015.

http:www.uel.br/revistas/infoprof/ 
Maria Cristina de Paiva Ribeiro

Projeto de dinamização da coleção de obras raras da Biblioteca do TRF $2^{\circ}$

Região

\section{Titulo}

Obras colección de estimulación proyecto raro TRF biblioteca segundo región

\section{Resúmen}

Introducción: Una biblioteca necesita mantener un espacio con obras relevantes para la mejora del conocimiento, el cual será organizado y preservado para la posteridad a través de una metodología específica y efectiva criterios de conservación. Esta es la intención del Proyecto de Racionalización de Organización y Tratamiento Técnico de Colección de Libros Raros Biblioteca Tribunal Regional Federal de la $2^{\mathrm{a}}$ Región (TRF2).

Proposito: Para enfocar la importancia de mantener la Colección de Libros Raros TRF2 de los procedimientos requeridos por el Proyecto.

Metodología: Estudio descriptivo y bibliográfico basado en una investigación bibliográfica de base teórica en búsquedas en Internet de las bibliotecas judiciales que mantienen colecciones de libros raros y estudio y análisis del Proyecto.

Resultados: El perfil del patrimonio cultural del libro raro es en la acción del autor como un agente de la Historia y la Memoria. La mayoría de las bibliotecas sucursales judiciales tienen colecciones de libros raros, lo que refuerza la implementación del Proyecto.

Conclusiones: EI $\mathrm{P}$ royecto permitió el desarrollo de criterios orientadores rareza bibliográfica, el establecimiento de una política de catalogación apropiada a la descripción de los libros raros y se colocan en la necesidad de mantener un espacio para la perpetuación de la Ley de la memoria por la producción jurídica representada en Colección de Libros Raros.

Palabras clave: Diseño Dinámico y el Tratamiento Técnico de la Colección de Libros Raros TRF Biblioteca Segunda Región de la Organización; Libro Raro; Biblioteca de Derecho; Organización del Conocimiento.

Recebido em: 15/03/2015

Aceito em: 10/09/2015 